Publ. RIMS, Kyoto Univ.

13 (1977), 167-172

\title{
Point Spectra of Non-Singular Flows
}

By

\author{
Motosige OSIKAWA*
}

\begin{abstract}
An example is given of a family of non-singular transformations whose point spectra are uncountable.
\end{abstract}

\section{$\S$ 1. Definition of the Point Spectrum}

Let $(X, \mathscr{Z}, P)$ be a Lebesgue measure space and let $\left\{T_{t} ; t \in \mathbb{R}\right\}$ be a one-parameter group of null-measure-preserving transformations of $X$ onto itself, where the map $X \times \mathbb{R} \rightarrow X\left((x, t) \rightarrow T_{t} x\right)$ is measurable. The non-singular flow $\left\{T_{t} ; t \in \boldsymbol{R}\right\}$ is said to be ergodic if every measurable function $f$ with $f\left(T_{t} x\right)=f(x)$ a.e.x for each $t \in \mathbb{R}$ is constant a.e.. A real number $\theta$ is called an element of the point spectrum of $\left\{T_{t} ; t \in \mathbb{R}\right\}$ if there exists a non-zero measurable function $\varphi$ with $\varphi\left(T_{t} x\right)=e^{i \theta t} \varphi(x)$ a.e.x for each $t \in \mathbb{R}$. If $\left\{T_{t} ; t \in \mathbb{R}\right\}$ is ergodic we may consider $|\varphi(x)|$ $=1$. We denote by $\operatorname{S} p\left(\left\{T_{t}\right\}\right)$ the point spectrum of $\left\{T_{t} ; t \in \mathbb{R}\right\}$. It is well known that if $\left\{T_{t} ; t \in \mathbb{R}\right\}$ has a finite invariant measure equivalent to $P \operatorname{Sp}\left(\left\{T_{t}\right\}\right)$ is $\{0\}$ or a countable subset of the set of all real numbers.

\section{§ 2. AC. Flow}

For every integer $n \geqq 1$ let $\Omega_{n}$ be the $l_{n}$-point set $\left\{0,1, \cdots, l_{n}-1\right\}$ for some integer $l_{n} \geqq 2, \mathfrak{B}_{n}$ be the algebra consisting all subsets of $\Omega_{n}$ and $G_{n}$ be the group of all permutations of $\Omega_{n}$. Let $\Omega$ be the product $\prod_{n=1}^{\infty} \Omega_{n}$ of the $\Omega_{n}, n \geqq 1$. For a point $\omega$ of $\Omega, \omega_{n}$ means the $n$-th coordinate of $\left(\omega\right.$. We may consider $\mathfrak{B}_{n}$ as an algebra on $\Omega$, denote by $\mathfrak{B}$ the algebra

Communicated by H. Araki, September 3, 1976.

* Department of Mathematics, College of General Education, Kyushu University, Fukuoka 810, Japan. 
generated by $\bigcup_{j=1}^{\infty} \mathfrak{B}_{j}$, and by $\mathfrak{B}_{n}^{*}$ the $\sigma$-algebra generated by $\bigcup_{j=1}^{n} \mathfrak{B}_{j}$. We may consider $G_{n}$ as a transformation group on $\Omega$, denote by $G$ the group generated by $\bigcup_{j=1}^{\infty} G_{j}$ and by $G_{n}^{*}$ the group generated by $\bigcup_{j=1}^{n} G_{j}$.

We define a transformation $T$ of $\Omega$ onto itself as follows: if $\omega_{j}=l_{j}$ -1 for $j=1,2, \cdots, n-1$ and $\omega_{n} \leqq l_{n}-2$ then $(T \omega)_{j}=0$ for $j=1,2, \cdots$, $n-1,(T \omega)_{n}=\omega_{n}+1$ and $(T \omega)_{j}=\omega_{j}$ for $j=n+1, n+2, \cdots . \quad T$ is called the adding machine with measure $\mu$ if $\mu(A)=0$ implies $\mu(T A)=0$.

For given numbers $X_{n}(k) \in \mathbb{R}, k=0,1, \cdots, l_{n}-1, n=1,2, \cdots$ such that $X_{n}(0)=0$ and $X_{n}(k+1)>X_{n}(k)+\sum_{j=1}^{n-1} X_{j}\left(l_{j}-1\right), k=0,1, \cdots, l_{n}-2$, $n=1,2, \cdots$, we define, if $\omega_{j}=l_{j}-1$ for $j=1,2, \cdots, n-1$ and $\omega_{n}=k \leqq l_{n}-2$, $f(\omega)=X_{n}(k+1)-X_{n}(k)-\sum_{j=1}^{n-1} X_{j}\left(l_{j}-1\right)$. We may consider $X_{n}(\cdot)$ as a $\mathfrak{B}_{n}$-measurable function defined on $\Omega$ and we have $f(\omega)=\sum_{n=1}^{\infty}\left(X_{n}(T \omega)\right.$ $\left.-X_{n}(\omega)\right)>0$. Consider the following flow built under a function $f$. Let $X$ be the set of all points $(\omega, s)$ of the product space $\Omega \times \mathbb{R}$ with $0 \leqq s<f(\omega), \mathscr{F}$ be the restriction of the product $\sigma$-algebra $\mathfrak{B} \times \mathfrak{B}_{\boldsymbol{R}}$ to $X$ and $P$ be the restriction of the product measure $\mu \times e^{-s} d s$ to $X$, where $d s$ is the uniform measure defined on the Borel field $\mathfrak{B}_{\boldsymbol{R}}$ of $\mathbb{R}$. A flow $\left\{T_{t}, t \in \boldsymbol{R}\right\}$ is defined on $(X, \mathscr{F}, P)$ as follows.

$$
T_{t}(\omega, s)=\left\{\begin{array}{c}
\left(\left(T^{-n} \omega, s+t+\sum_{j=1}^{n} f\left(T^{-j} \omega\right)\right)\right. \\
\text { if }-\sum_{j=1}^{n} f\left(T^{-j} \omega\right) \leqq s+t<-\sum_{j=1}^{n-1} f\left(T^{-j} \omega\right) \\
(n=1,2, \cdots) \\
\left(\left(T^{n} \omega, s+t-\sum_{j=1}^{n-1} f\left(T^{j} \omega\right)\right)\right. \\
\text { if } \sum_{j=0}^{n-1} f\left(T^{j} \omega\right) \leqq s+t<\sum_{j=0}^{n} f\left(T^{j} \omega\right) \\
(n=0,1, \cdots)
\end{array}\right.
$$

We call this $\left\{T_{t}, t \in \boldsymbol{R}\right\}$ the AC-flow determined by $T, \mu$ and $\left\{X_{n}(\cdot)\right\}$. (AC for Adding machine and Ceiling function, or perhaps for Alain Connes.)

\section{$\S 3$. Point Spectra of AC-Flows}

Theorem. Let $\left\{T_{t} ; t \in \boldsymbol{R}\right\}$ be the $A C$-flow determined by $T$, $\mu$ and 
$\left\{X_{n}(\cdot)\right\}$

(a) If there exists a sequence $\left\{C_{n}\right\}$ of real numbers such that $\exp \left\{i \theta \sum_{j=1}^{n}\left(X_{j}(\omega)-C_{j}\right)\right\}$ converges a.e. as $n \rightarrow \infty$, then $\theta \in \operatorname{Sp}\left(\left\{T_{t}\right\}\right)$.

(b) If $\mu$ is an infinite product of measures $\mu_{n}$ on $\Omega_{n}, n=1,2, \cdots$ with $\mu_{n}(\{k\})>0$, then $T$ is ergodic and the converse of $(a)$ is true.

Proof. It is easy to see that $\theta \in \mathrm{S} p\left(\left\{T_{t}\right\}\right)$ iff there exists a nonzero measurable function $\varphi(\omega)$ with

$\hat{w}$

$$
\varphi(T \omega)=e^{i \theta f(\omega)} \varphi(\omega) .
$$

If $\varphi(\omega)=\exp \left\{i \theta \sum_{j=1}^{\infty}\left(X_{j}(\omega)-C_{j}\right)\right\}$ exists, it satisfies $\hat{\jmath}$. Let $\varphi(\omega)$ be a non-zero function satisfying $\hat{\zeta}$, we have

$$
\exp \left\{i \theta \sum_{j=1}^{\infty}\left(X_{j}\left(T^{k} \omega\right)-X_{j}(\omega)\right)\right\}=\frac{\varphi\left(T^{k} \omega\right)}{\varphi(\omega)}, \quad k=0,1,2, \cdots .
$$

Since for any $g \in G_{n}^{*}$ and for a.e $\omega \in \Omega$, there exists an integer $k(g, \omega)$ such that $g \omega=T^{k(g, \omega)} \omega$,

$$
\exp \left\{i \theta \sum_{j=1}^{n}\left(X_{j}(g \omega)-X_{j}(\omega)\right)\right\}=\frac{\varphi(g \omega)}{\varphi(\omega)} \text {, a.e. } \omega \text { for each } g \in G_{n}{ }^{*}
$$

Since $\varphi_{n}^{*}(\omega)=\varphi(\omega) \exp \left\{-i \theta \sum_{j=1}^{n} X_{j}(\omega)\right\}$ is a $G_{n}^{*}$-invariant function and since $\mathfrak{B}_{n}$ 's are mutually independent $\sigma$-algebras on the assumption of (b), we have

$$
\lim _{n \rightarrow \infty} \exp \left\{i \theta \sum_{j=1}^{n} X_{j}(\omega)\right\} E\left(\varphi_{n}^{*}(\omega)\right)=\lim _{n \rightarrow \infty} E\left(\left(\varphi(\omega) / \mathcal{B}_{n}^{*}\right)=\varphi(\omega) .\right.
$$

Hence there exists a sequence $\left\{C_{n}\right\}$ of real numbers such that $\exp \left\{i \theta \sum_{j=1}^{n}\left(X_{j}(\omega)-C_{j}\right)\right\}$ converges a.e. as $n \rightarrow \infty$. (cf. [2])

\section{§4. Example 1}

Put $\Omega_{n}=\{0,1\}, \mu_{n}(0)=\frac{1}{1+\lambda}, \mu_{n}(1)=\frac{\lambda}{1+\lambda}$ and $X_{n}(0)=0, X_{1}(1)$ $=2^{n} a, n=1,2, \cdots$ for some $0<\lambda \leqq 1$ and some $a$ in $\mathbb{R}$. Let $\left\{T_{t} ; t \in \boldsymbol{R}\right\}$ be the AC-flow determined by the product measure $\mu=\prod_{n=1}^{\infty} \mu_{n}$ and the 
$\left\{X_{n}(\cdot)\right\}$. Then $\mathrm{S} p\left(\left\{T_{t}\right\}\right)=\frac{2 \pi}{a} \times\{2$-adic rational numbers $\} . \quad$ In fact since for any 2 -adic rational number $\theta \exp \left\{i \frac{2 \pi}{a} \theta X_{n}(\omega)\right\}=1$ for sufficiently large $n, \exp \left\{i \frac{2 \pi}{a} \theta \sum_{n=1}^{\infty} X_{n}(\omega)\right\}$ converges a.e.. Try next to assume that $\exp \left\{i \frac{2 \pi}{a} \theta \sum_{n=1}^{\infty}\left(X_{n}(\omega)-C_{n}\right)\right\}$ converges a.e. for some not 2adic rational number $\theta$ and some sequence $\left\{C_{n}\right\}$ of real numbers. Put $E=\left\{\omega \in \Omega ; \exp \left(i \frac{2 \pi}{a} \theta\left(X_{n}(\omega)-C_{n}\right)\right)\right.$ converges to 1 as $\left.n \rightarrow \infty\right\} ;$ we have $\mu(E)=1$. Looking at fractional parts of $\theta 2^{n}$, one sees there exists an infinite subset $N_{1}$ of positive integers such that $\left|\exp \left\{i 2 \pi \theta 2^{n}-1\right\}\right|>\sqrt{2}$, $n \in N_{1}$. Since, from the Borel-Cantelli lemma, there exist an $\omega$ in $E$ and an infinite subset $N_{2}$ of $N_{1}$ such that $\omega_{n}=1$ for $n \in N_{2}$, $\lim _{\substack{n \in N_{2} \\ n \rightarrow \infty}} \exp \left(i 2 \pi \theta 2^{n}\right) \exp \left(-i \frac{2 \pi}{a} \theta C_{n}\right)=1$. Again from the Borel-Cantelli lemma there exist an $\omega$ in $E$ and an infinite subset $N_{3}$ of $N_{2}$ such that $\omega_{n}=0$ for $n \in N_{3}$, so $\lim _{\substack{n \in N_{3} \\ n \rightarrow \infty}} \exp \left(-i \frac{2 \pi}{a} \theta C_{n}\right)=1$. This is a contradiction and thus $\frac{2 \pi}{a} \theta$ is not in $\operatorname{Sp}\left(\left\{T_{t}\right\}\right)$. (cf. [2])

\section{§5. Example 2 (with uncountable spectra)}

Put $M_{1}=2, M_{n}=2^{n} M_{n-1}(n=2,3, \cdots)$. For any real number $\theta$ there exists a unique sequence $\left\{l_{1}{ }^{\prime}, l_{2}{ }^{\prime}, \cdots, l_{n}{ }^{\prime}, \cdots\right\}$ of integers such that $-2^{n-1}$ $\leqq l_{n}^{\prime} \leqq 2^{n-1}, n=2,3, \cdots$ and $-\frac{1}{2 M_{n}}<\theta-\left(\frac{l_{1}^{\prime}}{M_{1}}+\frac{l_{2}^{\prime}}{M_{2}}+\cdots+\frac{l_{n}{ }^{\prime}}{M_{n}}\right) \leqq \frac{1}{2 M_{n}}, n$ $=1,2, \cdots$. For any sequence $\left\{l_{1}, l_{2}, \cdots, l_{n}, \cdots\right\}$ of integers with $\left|l_{n}\right| \leqq 2^{n-2}$ for sufficiently large $n$, the unique sequence $\left\{l_{1}{ }^{\prime}, l_{2}{ }^{\prime}, \cdots, l_{n}{ }^{\prime}, \cdots\right\}$ of integers determined by a real number $\frac{l_{1}}{M_{1}}+\frac{l_{2}}{M_{2}}+\cdots+\frac{l_{n}}{M_{n}}+\cdots \quad$ satisfies $\quad l_{n}{ }^{\prime}=l_{n}$ for sufficiently large $n$, because $\mid \frac{l_{n}}{M_{n}}+\frac{l_{n+1}}{M_{n+1}}+\cdots<\frac{1}{2 M_{n-1}}$. Hence the set of all real numbers whose uniquely determined sequence $\left\{l_{1}{ }^{\prime}, l_{2}{ }^{\prime}, \cdots\right.$, $\left.l_{n}{ }^{\prime}, \cdots\right\}$ satisfies $\sum_{n=1}^{\infty}\left(\frac{l_{n}{ }^{\prime}}{2^{n}}\right)^{2}<+\infty$ is the set of all real numbers $\frac{l_{1}}{M_{1}}+$ $\frac{l_{2}}{M_{2}}+\cdots+\frac{l_{n}}{M_{n}}+\cdots$ determined by a sequence $\left\{l_{1}, l_{2}, \cdots, l_{n} \cdots\right\}$ with $\sum_{n=1}^{\infty}$ $\left(\frac{l_{n}}{2^{n}}\right)^{2}<+\infty$. We denote by $\mathscr{M}$ the latter set. It is easy to see that $\mathscr{M}$ is an uncountable subgroup of the group of real numbers and that 
the complement of $\mathscr{M}$ is also uncountable.

Put $\Omega_{n}=\{0,1\}, X_{n}(0)=0, X_{n}(1)=M_{n} a, n=1,2, \cdots$ for some $a$ in $\mathbb{R}$ and let $\left\{T_{t} ; t \in \mathbb{R}\right\}$ be the AC-flow determined by the adding machine with an arbitrary measure $\mu$ and the $\left\{X_{n}(\cdot)\right\}$. For a real number $\theta$ $=\frac{l_{1}}{M_{1}}+\frac{l_{2}}{M_{2}}+\cdots+\frac{l_{n}}{M_{n}}+\cdots$ denote by $Y_{n}\left(\omega_{n}\right)$ the fractional part of $\frac{\theta}{a} X_{n}(\omega)$, $n=1,2, \cdots, \quad$ we have $Y_{n}(0)=0 \quad$ and $\quad Y_{n}(1)=M_{n}\left(\frac{l_{n+1}}{M_{n+1}}+\frac{l_{n+2}}{M_{n+2}}+\right.$ $\cdots), n=1,2, \cdots$. All real numbers $\frac{2 \pi}{a}\left(\frac{l_{1}}{M_{1}}+\frac{l_{2}}{M_{2}}+\cdots+\frac{l_{n}}{M_{n}}+\cdots\right)$ with $\left|l_{n}\right| \leqq 1, n=1,2, \cdots$ are in $\mathrm{S} p\left(\left\{T_{t}\right\}\right)$, since $\sum_{n=1}^{\infty}\left|Y_{n}(\cdot)\right| \leqq \sum_{n=1}^{\infty} M_{n}\left(\frac{1}{M_{n+1}}+\right.$ $\left.\frac{1}{M_{n+2}}+\cdots\right) \leqq \sum_{n=1}^{\infty} \frac{1}{2^{n}}<+\infty$

If $\mu$ is an infinite product of measures $\mu_{n}$ on $\Omega_{n}, n=1,2, \cdots$ such that $\mu_{n}(0)=\frac{1}{1+\lambda}, \mu_{n}(1)=\frac{\lambda}{1+\lambda}, n=1,2, \cdots,(0<\lambda \leqq 1)$, we have $\operatorname{S} p\left(\left\{T_{t}\right\}\right)$ $=\frac{2 \pi}{a} \mathscr{M}$. In fact $\exp \left\{2 \pi i \sum_{n=1}^{\infty}\left(Y_{n}(\omega)-C_{n}\right)\right\}$ converges a.e. for some seqence $\left\{C_{n}\right\}$ of real numbers iff $\sum_{n=1}^{\infty} V\left(Y_{n}(\omega)\right)<+\infty$, iff $\sum_{n=1}^{\infty} M_{n}^{2}\left(\frac{l_{n+1}}{M_{n+1}}+\frac{l_{n+2}}{M_{n+2}}+\cdots\right)^{2}<+\infty$ and iff $\sum_{n=1}^{\infty} M_{n}^{2}\left(\frac{l_{n+1}}{M_{n+1}}\right)^{2}<+\infty$.

\section{§6. Remarks Related to the Weak Equivalence Theory of Non-Singular Transformations}

(1) J. Woods [5] constructed ITPFI factors with an uncountable $\rho$-set. A. Connes [1] constructed an adding machine with infinite product measure whose associated flow was the AC-flow determined by the adding machine and some $\left\{X_{n}(\cdot)\right\}$. Using his method we can construct an adding machine with infinite product measure whose associated flow is the AC-flow of Example 2. This has uncountable T-set $\frac{2 \pi}{a} \mathscr{M}$. (cf. [3] and [4])

(2) We may take as parameter set the set $\mathbb{Z}$ of all integers instead of $\mathbb{R}$ as in this paper. In this case we would say AC-transformation instead of AC-flow. If an adding machine $T$ has no $\sigma$-finite invariant measure equivalent to $\mu$, every AC-transformations determined by $T$ and some ceiling is weakly equivalent to $T$. The AC-transformations of 
Example 1 for different prime numbers $a$ have mutually different spectra. So they are weakly equivalent (of type $\mathrm{III}_{\lambda}, 0<\lambda<1$ ), but not equivalent.

\section{Acknowledgement}

I would like to express my thanks to W. Krieger for his kind hospitality at the University of Heidelberg where this work was completed and to P. D. F. Ion for some useful discussions.

\section{References}

[1] Connes, A., On the hierarchy of W. Krieger, Illinois J. Math., 19 (1975), 428-432.

[2] Hamachi, T., Oka, Y. and Osikawa, M., A classification of ergodic non-singular transformation groups, Mem. Fac. Sci. Kyushu Univ., 18 (1974), 113-133.

[3] Hamachi, T., Oka, Y. and Osikawa, M., Flows associated with ergodic non-singular transformation groups, Publ. RIMS, Kyoto Univ., 11 (1975), 31-50.

[4] Krieger, W., On ergodic flows and the isomorphism of factors, to appear in Math. Ann.

[5] Woods, E. J., The classification of factors is not smooth, Canad. J. Math., 15 (1973), 96-102. 